\title{
Le bois-énergie dans la tempête, entre innovation et captation? Les nouvelles ressources de la forêt landaise
}

\author{
Vincent Banos ${ }^{1, *}$ et Jeoffrey Dehez ${ }^{2}$ \\ ${ }^{1}$ Géographe, Irstea, unité ETBX, Cestas, France \\ 2 Économiste, Irstea, unité ETBX, Cestas, France
}

Reçu le 30 avril 2015. Accepté le 6 avril 2017

Le bois-énergie joue un rôle important dans la course aux énergies renouvelables. À partir du cas du massif landais, les auteurs analysent la mobilisation accrue de la ressource forestière qui s'opère à travers l'exploitation des souches d'arbres. Cette extension de la ressource forestière et cet accroissement du potentiel bois-énergie sont permis par des innovations techniques qui mêlent de façon indissociable aspects technologiques et sociaux. Dans ce massif forestier, essentiellement privé et intensément exploité, l'industrie papetière porte ce projet en renégociant les rapports avec les autres acteurs (propriétaires, exploitants, transformateurs, etc.) en fonction de ses stratégies de développement ; l'utilisation des politiques publiques de soutien mises en place est centrale dans cette dynamique et les questions de rentabilité économique des souches en deviennent presque secondaires. Les fenêtres d'opportunité ouvertes par les tempêtes successives qui ont frappé le massif forestier sont exploitées pour accélérer et orienter les négociations, laissant de côté les questions de stockage du carbone et de maintien de la fertilité des sols. Dans ce cas, la transition énergétique pourrait se traduire par un renforcement des industries dominantes et une intensification de la pression sur les ressources et les milieux.

La Rédaction

Résumé - En France, les souches forestières font désormais partie des gisements de biomasse ligneuse identifiés pour contribuer à la transition énergétique. Mais la récolte de cette partie basse et souterraine des arbres est pour l'instant limitée à la forêt cultivée des Landes de Gascogne. Cet article se propose d'analyser les ressorts de cette innovation territoriale en mobilisant un cadre analytique et méthodologique issu du champ «sciences, technologies et société (STS)». Les auteurs montrent ainsi que la valorisation de cette nouvelle ressource est portée par des industries de la filière bois-papier soucieuses de préserver leurs matières premières usuelles et d'ajuster le développement des politiques de soutien aux énergies renouvelables. Ils soulignent aussi que ce travail d'innovation a bénéficié du concours inattendu mais décisif de deux tempêtes majeures (1999 et 2009). Leur analyse dévoile in fine la construction d'une politique « locale» du bois-énergie qui, orientée vers le renforcement d'industries déjà bien établies et l'intensification des pratiques sylvicoles, questionne les potentialités associées à la transition énergétique.

Mots-clés : bois-énergie / ressources naturelles / matérialités / innovation / système sociotechnique / Aquitaine

\begin{abstract}
In the eye of the storm: harvesting tree stumps and the energy transition. Since 2009 wood stumps are considered as one of the recognized sources of biomass to be used in the process of national energy transition in France. So far, stump harvesting has only been implemented in the Landes area in the southwestern part of the country. The aim of this paper is to identify the mechanisms underpinning this innovation. For this we used an analytical framework inspired by Science and Technology Studies. We show how the local pulp and paper industry has ensured the safety of its own supply agendas by promoting adjustments to renewable energy policies. These same firms took advantage of the two major storms which occurred in 1999 and 2009. Our study traces the development of a "local"
\end{abstract}

\footnotetext{
*Auteur correspondant : vincent. banos@irstea.fr
} 
wood energy policy aimed at consolidating well established firms and intensifying silvicultural management practices, which raise a number of questions regarding the true potential for energy transition gained from such a system.

Keywords: wood biomass / natural resources / materiality / innovation / sociotechnical system / Aquitaine

Représentant plus de $40 \%$ des énergies renouvelables (EnR) produites en 2014, le bois-énergie (BE) est la première source d'EnR, loin devant l'hydraulique $(26 \%)$, et un élément incontournable des politiques de transition énergétique menées en France (Carrier et al., 2015). Pour atteindre l'objectif de $32 \%$ d'EnR en $2030^{1}$, l'État et les acteurs de la politique forestière se sont d'ailleurs prononcés en faveur d'une augmentation significative de la mobilisation des ressources ligneuses : +12 millions de $\mathrm{m}^{3}$ à l'horizon 2026, soit un accroissement de plus de $20 \%$ des prélèvements actuels (MAAF, 2016). Au-delà des chiffres, ce volontarisme s'accompagne également d'une extension des types de ressources explorées. Depuis 2009, les souches forestières, c'est-à-dire la partie basse et souterraine des arbres, sont ainsi intégrées aux inventaires sur les gisements de biomasse mobilisables pour des usages énergétiques (Colin et al., 2009). Qualifiée « d'annexe», au même titre que les vignes, les vergers ou les arbres urbains, cette ressource présenterait néanmoins l'avantage d'être inexploitée (Colin et al., 2009). Mais si elle est une réalité en Scandinavie, la récolte des souches est controversée dans de nombreux pays en raison de ses conséquences possibles sur la fertilité des sols et le stockage du carbone (Walmsley et Godbold, 2009) et, dans le cas de la France, limitée à la forêt des Landes de Gascogne (Landmann et Nivet, 2013).

Questionner l'émergence d'une ressource ${ }^{2}$ offre l'opportunité de décaler le regard sur une transition énergétique encore très souvent pensée, tant au niveau scientifique que politique ${ }^{3}$, en termes de développement technologique. En se focalisant sur le rôle déterminant de l'innovation technologique et des changements institutionnels nécessaires à sa diffusion (Geels, 2002), ces réflexions tendent à réduire les EnR (soleil, vent, bois,

\footnotetext{
${ }^{1}$ Loi de «Transition énergétique pour une croissance verte » (juillet 2015).

${ }^{2}$ Ce travail s'inscrit dans le cadre de l'ANR COLLENER (2012-2015) et a fait l'objet d'une première communication : Banos V., Dehez J., 2013. "The evolution of wood biomass projects in Aquitaine: is there more than just trees?" Communication au colloque RGS-IBG Annual International Conference, session "Emerging socio-technical collectives and new geographies of the energy transition", 27-30 août, Londres.

${ }^{3}$ «La biomasse, c'est la nature au service de la technologie » (ministère de l'Écologie, du Développement durable et de l’Énergie, colloque national Biomasse, juillet 2014).
}

etc.) à des gisements dotés d'un potentiel énergétique intrinsèque. Ce faisant, elles minimisent l'hétérogénéité du monde biophysique ainsi que les usages et les valeurs, les organisations collectives et les droits de propriété auxquels ces entités naturelles sont rattachées (Labussière et Nadai, 2015 ; Lawhon et Murphy, 2011). Or, les sciences sociales ont largement démontré que les ressources naturelles n'existent pas en soi mais adviennent après un processus de requalification et de transformation matérielle de la nature qui engage un travail culturel, politique et économique (De Gregori, 1987 ; Fitzsimmons, 1989). Poussant cette perspective relationnelle, la Political ecology s'est notamment attachée à montrer comment les changements d'usages de la nature, toujours situés dans des contextes géographique et historique particuliers, impliquaient des luttes de pouvoir et pouvaient générer des asymétries socioéconomiques tant en termes de contrôle des ressources que d'accumulation des richesses (Benjaminsen et Svarstad, 2009; Castree et Braun, 2001; Robbins, 2004). Sans perdre son ambition initiale, ce courant de pensée s'est plus récemment ouvert au champ des «sciences, technologies et société (STS)» en considérant notamment le rôle actif des objets et des processus biophysiques dans la co-construction des relations socioécologiques (Bassett et Peimer, 2015; Perkins, 2007; Robbins, 2007). La démarche proposée dans cet article s'inscrit dans cette perspective puisque, tout en partageant le questionnement porté par la Political ecology, nous mobilisons un cadre analytique et méthodologique issu des STS, et plus précisément sa théorie de l'innovation. Ce croisement semble intéressant pour interroger les potentialités associées à la transition énergétique. A priori mieux réparties et plus accessibles que les énergies fossiles, les EnR seraient en effet propices à une redistribution des pouvoirs, des risques et des richesses (Labussière et Nadai, 2015; Raineau, 2011; Rumpala, 2013).

Le $\mathrm{BE}$ est révélateur de la situation en trompe l'œil des EnR. Si cette énergie primaire est maîtrisée depuis longtemps, ses technologies évoluent fortement avec le développement d'équipements collectifs et industriels orientés vers la production de chaleur mais aussi d'électricité. De même, si les ressources forestières peuvent apparaître abondantes et locales (IFN, 2005), leur accessibilité dépend des configurations géographiques et de la manière dont elles sont déjà sollicitées par la filière bois-papier. La concurrence peut être d'autant plus vive que les principaux gisements de $\mathrm{BE}$, c'est-à-dire les bois d'éclaircies, les rémanents de l'exploitation 


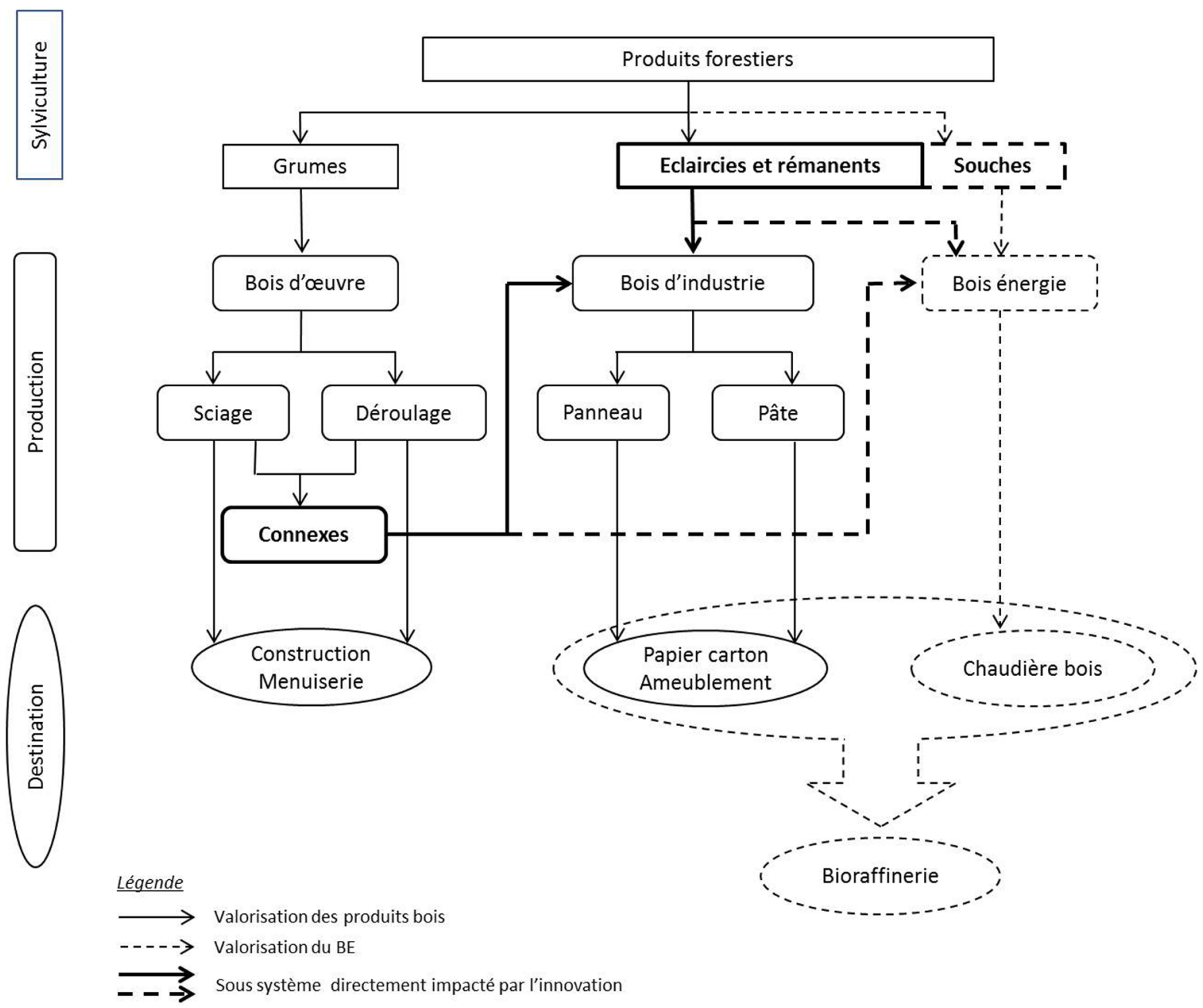

Fig. 1. Illustration des interdépendances au sein du système sociotechnique landais.

Source : Dehez J., Banos V., 2016.

forestière et les déchets de scieries, constituent également la matière première des industries de la trituration et en particulier des papetiers (Fig. 1). Or, ceux-ci contribuent activement à structurer les espaces forestiers, notamment dans un massif des Landes de Gascogne mis en valeur au $\mathrm{XIX}^{\mathrm{e}}$ siècle et aujourd'hui présenté comme la plus grande forêt cultivée d'Europe (environ un million d'hectares de pin maritime). Dans cet article, nous défendons ainsi l'hypothèse que la valorisation des souches est une innovation portée par les papetiers landais pour ajuster le développement des politiques de transition énergétique et que, dans ce processus complexe, ils ont notamment pu s'appuyer sur des événements imprévus tels que les tempêtes.

La première partie revient sur les principes analytiques et méthodologiques de la théorie de l'innovation ainsi que sur les protagonistes et les dynamiques d'une forêt des Landes de Gascogne saisie ici comme un système sociotechnique. Les deux parties suivantes sont consacrées à la description des ressorts ayant permis la transformation des souches en ressource biomasse en insistant tout $\mathrm{d}$ 'abord sur la genèse de cette innovation, à l'intersection de préoccupations sectorielles et du développement des dispositifs de soutien aux EnR, avant de s'attarder sur le rôle des tempêtes Martin (en 1999) et Klaus (en 2009).

\section{Déplier «les tissus sans couture », retour sur une théorie de l'innovation}

Contestant la vision d'une innovation se diffusant de manière linéaire grâce aux qualités intrinsèques de la technologie proposée (sa rentabilité, sa maturité, etc.), les tenants de l'approche STS développent dans les années 1980 une théorie alternative. Celle-ci part du principe que l'innovation est fondamentalement une navigation en eau trouble qui nécessite de tenir ensemble le social, le technique et l'économique (Akrich, 1989; Akrich et al., 1988; Callon, 1986). Si ce cadre d'analyse a largement pénétré le champ des sciences sociales, la formulation persistante des enjeux de l'innovation en termes de 
rentabilité économique ou d'acceptabilité sociale (Barbier et Nadai, 2015) invite à revenir sur ses apports. Pour ce faire, nous retenons trois caractéristiques essentielles à notre démarche.

Premièrement, alors que le modèle diffusionniste suppose une séparation irrémédiable entre l'innovation et son environnement socioéconomique, l'approche STS considère que "l'innovation se produit dans un environnement qui se modifie en même temps que le projet et avec lui» (Callon, 1999). Non seulement l'innovateur doit s'adapter à des marchés et des politiques publiques qui évoluent souvent de manière imprévisible mais, de plus, l'objet technique et le monde dans lequel il est appelé à fonctionner se transforment mutuellement. Cette idée d'une innovation indissociablement sociale et technique est au fondement de la notion de système sociotechnique proposée par Akrich (1989) dans son article séminal sur le développement d'une innovation permettant de fabriquer et valoriser un combustible à partir de tiges de coton au Nicaragua.

Deuxièmement, l'approche STS considère que le succès d'une innovation dépend moins de sa rentabilité, qui n'est au mieux qu'une conséquence heureuse du projet, que de sa capacité à entraîner les acteurs qui à un moment ou un autre vont influencer son destin: «L'innovation, c'est l'art d'intéresser un nombre croissant d'alliés » (Akrich et al., 1988). Pour parvenir à mobiliser et enrôler ces acteurs, dont rien ne garantit a priori qu'ils se plient au scénario imaginé, l'innovateur doit construire des dispositifs d'intéressement qui permettront à l'innovation d'être en prise avec ce qui a été problématisé comme son environnement futur (Akrich, 1989). Il doit démontrer les bénéfices attendus de son projet, avant même que le marché n'existe, et stabiliser les alliances de manière à éviter que les acteurs ne se tournent vers d'autres solutions; en somme ériger son projet en point de passage obligé (Callon, 1986). Si ce travail d'assemblage suppose des ajustements réciproques, il accorde néanmoins beaucoup de pouvoir à ceux qui sont chargés de négocier le projet pour le mettre en forme jusqu'à ce qu'il se constitue en marché : «Être porte-parole, c'est parler au nom d'autrui mais c'est surtout faire taire autrui »(Callon, 1999). Cette vision paraît donc surtout adaptée aux situations où le travail de l'innovateur consiste davantage à transformer qu'à créer un système sociotechnique; ce qui est bien le cas de la forêt des Landes de Gascogne.

La troisième caractéristique consiste à reconnaître la capacité des objets et des processus biophysiques à lier ou à séparer les acteurs et donc à peser sur la trajectoire de l'innovation. Cette contribution doit s'envisager à deux niveaux. Tout d'abord, le travail consistant à définir, objectiver et transformer les qualités d'un objet, via des opérations de "traduction», est au cœur des relations entre acteurs. Si cette attention accordée aux activités métrologiques et aux processus de qualificationrequalification n'est pas propre à l'approche STS, l'intérêt du cadre d'analyse proposé est qu'il étend ses ramifications jusqu'aux échanges marchands ${ }^{4}:$ « Le prix doit être considéré comme une qualité au même titre que les autres qualités » (Callon, 2009). Ensuite, de par leur matérialité et leurs dynamiques propres, les objets ou phénomènes biophysiques influent sur le travail de l'innovateur. Vecteurs d'une mise en ordre du monde ou sources de rupture inattendue, ils ont la capacité de faciliter, d'accélérer ou, au contraire, de contrecarrer le cours de l'action (Bakker et Bridge, 2006; Barbier et Trepos, 2007 ; Bennett, 2010).

En définissant in fine l'innovation comme la construction d'un système sociotechnique assemblant des acteurs et des entités hétérogènes, ce cadre d'analyse donne des clefs de lecture pour analyser les processus qui ont fini par rendre nécessaire et difficilement transformable une situation qui n'avait rien d'inévitable. Le paradoxe étant effectivement que si toute innovation suppose d'arbitrer des différends et d'écarter des possibles, elle doit, pour aboutir, apparaitre comme un assemblage stabilisé et naturalisé, comme un «tissu sans couture» (Callon, 1999).

\section{Matériel et méthode}

Le cadre analytique suppose, au niveau méthodologique, d'être attentif à la dynamique des objets et de restituer le cheminement suivi par les acteurs en évitant au maximum de faire intervenir des variables surplombantes définies a priori ou des éléments qui ne sont connus qu'en fin de parcours. Il s'agit donc de rendre compte des dispositifs et des argumentations mobilisés au moment où les décisions ont été prises. De même, on ne peut préjuger de l'importance déterminante de telle ou telle échelle géographique mais on doit essayer de suivre les innovateurs dans leur travail souvent pluriscalaire. Ces exigences posent quelques défis, notamment lorsque l'innovation s'étend sur plusieurs décennies. Issues d'un projet de recherche et d'une expertise collective ${ }^{5}$, les données utilisées sont tout d'abord composées d'une

\footnotetext{
${ }^{4}$ Dans cette perspective, « les marchés sont définis comme des dispositifs où s'affrontent et s'éprouvent des définitions contradictoires des biens et de leur qualification. Ces affrontements se résolvent dans des compromis qui prennent la forme de transactions donnant lieu à des paiements monétaires » (Callon, 2009).

${ }^{5}$ En plus des enquêtes menées dans cadre de l'ANR COLLENER (2012-2016), l'analyse mobilise des données recueillies lors de la prospective « Massif des Landes de Gascogne à l'horizon $2050 »(2009-2012)$.
} 
trentaine d'entretiens menés entre 2009 et 2014 auprès d'acteurs impliqués, à un moment ou un autre, dans l'innovation: industriels de la filière bois-papier landaise, propriétaires et opérateurs forestiers, énergéticiens, organismes de recherche et développement, collectivités territoriales et administrations publiques. Ce matériau a été complété par une analyse des dispositifs (réglementaires, économiques, etc.) mobilisés et des argumentaires développés dans différentes arènes (expertises, politiques publiques, médias, etc.). Même si les entretiens comportaient une dimension rétrospective, nous avons accordé une grande importance à cette analyse documentaire et mobilisé des archives afin de retracer le plus précisément possible le processus d'innovation (documents techniques, rapports d'études, presses professionnelles, articles scientifiques, etc.).

\section{Acteurs et dynamiques du système sociotechnique landais}

La forêt landaise peut être définie comme un système sociotechnique fluctuant construit autour d'un invariant : le pin maritime (Mora et Banos, 2014). Autrefois utilisée pour produire du goudron et du charbon, cette essence connaît son «âge d'or» avec le gemmage, avant d'être, depuis les années 1960, au cœur des interdépendances tissées entre les industries du bois d'œuvre (scieries, parquets, lambris, etc.) et de la trituration (papiers, panneaux). Dans ce modèle, chaque qualité de matière trouve une valorisation industrielle spécifique: tandis que les entreprises du bois d'œuvre (BO) transforment les grumes, les menus bois et les déchets de scieries composent le bois d'industrie (BI) mobilisé par la trituration (Fig. 1). L'essentiel de la récolte étant consommée localement, les propriétaires (privés à $92 \%)$ sont également enserrés dans cette organisation industrielle (Montouroy et Sergent, 2014). La diffusion rapide, à partir des années 1960, d'une ligniculture ou « culture du bois » (Maugé et Léonard, 1969) visant à accroître quantitativement et qualitativement la production de bois grâce à un travail du sol intensif (labour, semis, etc.) et une amélioration génétique des peuplements de pin maritime (rotations à 40-50 ans), témoigne de la force de ce système et des convergences qu'il a pu générer.

Ces interdépendances sont toutefois sous-tendues par des conflits d'intérêt et des déséquilibres. Alors que le secteur du sciage se caractérise par de petites structures aux faibles capacités d'investissement, les industries de la trituration sont dominées par de grands groupes positionnés sur des marchés mondiaux (Lévy et Belis-Bergouignan, 2011). Mais c'est d'abord par les volumes et le type de bois consommés que ces dernières exercent un certain pouvoir de coordination. Elles offrent des débouchés conséquents aux scieurs et aux propriétaires, tout en leur permettant d'écouler des déchets, dont le coût de traitement est important (Montouroy et Sergent, 2014). Les industries de la trituration sont également "attachées" à la forêt cultivée de pin maritime, d'une part parce que les fibres de cette essence offrent de bonnes propriétés mécaniques à leurs produits et d'autre part parce que ces entreprises lient leur compétitivité à l'accès à une ressource abondante. Au final, les relations apparaissent fondamentalement ambivalentes entre des propriétaires qui dénoncent l'aspect peu rémunérateur du marché de masse de la trituration et une industrie qui reproche aux sylviculteurs de conserver trop longtemps les bois sur pieds (logique de capitalisation). Afin de résoudre cette tension, les papetiers soutiennent depuis les années 1970 le développement de coopératives forestières chargées d'optimiser la production et d'encourager une sylviculture plus dynamique.

Si ces asymétries fragilisent le système sociotechnique landais, celui-ci a étendu ses ramifications bien au-delà du domaine sectoriel (Mora et Banos, 2014). Des liens étroits ont ainsi été tissés avec les organismes de recherche et de développement autour de cette forêt artificielle marquée par le progrès technique. De plus, malgré ses dissensions, la filière bois-papier sait faire front commun pour doter son modèle de profondes vertus territoriales et identitaires en jouant de son ancrage local et de son rôle structurant dans l'organisation socioéconomique du territoire (Mora et Banos, 2014). Cependant, l'irruption d'un nouvel usage, tel que celui du $\mathrm{BE}$, est à même de recomposer les équilibres existants.

\section{Les souches, reflets d'une adaptation sectorielle aux enjeux énergétiques}

Bien que certains industriels aiment à rappeler que les souches du pin maritime ont pu autrefois entrer dans la composition du goudron et du charbon, l'innovation menée n'a que peu à voir avec des pratiques marginales et depuis longtemps disparues. Sa raison d'être se trouve plutôt dans une double problématisation des enjeux énergétiques: préserver la ressource existante en pin maritime; instrumentaliser l'essor de la demande en bois-énergie pour intensifier la gestion forestière.

\section{Les chocs pétroliers, élément déclencheur ou faux départ?}

Les années 1970 marquent l'émergence de plusieurs programmes pilotes visant à évaluer les ressources supplémentaires dans les Landes de Gascogne (De Simiane et al., 1977). Ces explorations mobilisent de nombreux acteurs, dont des industries de la chimie et de la pétrochimie désireuses de jouer la carte du bois dans 
un contexte de crise pétrolière (ADISCO, 1983). Elles sont également fortement investies et orientées par des papetiers qui, grands consommateurs d'énergie thermique, sont aussi affectés par la fluctuation des prix du pétrole et cherchent à réduire leur dépendance aux énergies fossiles (Riedacker et Robin, 1987). De tels projets permettent également à ces derniers de se positionner comme «les acheteurs traditionnels» de la forêt landaise et de garder sous contrôle « les nouveaux consommateurs » (ADISCO, 1983), qui avant d'être des partenaires sont d'abord des concurrents potentiels. Enfin, ces explorations leur offrent l'occasion de mettre à l'agenda et de faire relayer une de leurs principales préoccupations, à savoir la nécessité d'accroître la production de $\mathrm{BI}$ : «Si les expériences en cours se révèlent satisfaisantes, il est permis de penser que les uns et les autres s'adapteront, y compris les propriétaires forestiers, qui seront, ainsi, incités à adopter une sylviculture plus intensive»(ADISCO, 1983). L'entremêlement des enjeux énergétiques et des problématiques propres à l'industrie de la trituration est également à l'origine des explorations menées à la même époque en Scandinavie (Hakkila, 2006; Polge, 1977).

Parmi les différents « déchets » identifiés, les souches sont rapidement définies comme "une des ressources nouvelles les plus massives et les plus accessibles» (ADISCO, 1983). Le gisement est estimé à environ $600000 \mathrm{~m}^{3}$ par an et ses promoteurs décrivent un milieu particulièrement propice à sa captation. Le sol plan et sableux des Landes de Gascogne faciliterait l'extraction et limiterait les risques d'érosion (De Simiane et al., 1977). Il est également avancé que le dessouchage améliorerait le travail du sol et permettrait de lutter contre les parasites qui se nourrissent du pourrissement des souches. Malgré ce dispositif d'intéressement séduisant pour les propriétaires forestiers, l'expérience se heurte à plusieurs obstacles et, en premier lieu, à l'objet lui-même. La forme de la souche, sa forte teneur en silice et le foisonnement racinaire alourdissent les opérations de transformation et contrecarrent les formes de valorisation envisagées par les industries de la trituration. Le sable use les couteaux utilisés dans la fabrication des panneaux, crible le papier de points et vitrifie les vieilles chaudières à grille (ADISCO, 1983). Toutes ces difficultés peinent à convaincre des propriétaires qui ont l'habitude de laisser les souches sur place (Maugé et Léonard, 1969). Ces derniers sont d'autant moins enclins à répondre aux attentes des industries de la trituration que le choc pétrolier de 1973 s'est accompagné d'une hausse importante des prix du BO : "L'intérêt bien compris des sylviculteurs est de faire le moins possible de $\mathrm{BI}$ et le maximum de $\mathrm{BO}^{6}{ } \gg$. De

\footnotetext{
${ }^{6}$ Forêt de Gascogne : Journal des sylviculteurs du Sud-Ouest, novembre 1973.
}

plus, les gains de productivité associés à la ligniculture et la fermeture d'une usine papetière sur le massif affaiblissent la portée du discours sur le déficit en BI. Enfin, les explorations engagées peinent à s'appuyer sur des dispositifs de soutien, en particulier dans le domaine énergétique. En France, il faut ainsi attendre 1994 pour voir émerger les premières incitations en faveur du BE. Essentiellement orientées vers l'installation de petites chaufferies collectives, elles n'affectent pas les équilibres de la filière gasconne et les projets lancés par les industries de la trituration sont mis en sommeil ou cantonnés à une logique d'autoconsommation jusqu'au début des années 2000 (Banos et Dehez, 2015).

\section{Une innovation relancée par les politiques de soutien à la production d'électricité renouvelable}

En 2001, l'Union européenne fixe l'objectif d'augmenter la part des EnR dans la production électrique à $21 \%$ d'ici 2010. Dans cette optique, elle autorise la mise en œuvre d'appels d'offre par les États et le rachat de l'électricité produite à un prix garanti. En France, cette mission est confiée à la Commission de régulation de l'énergie (CRE) qui, entre 2003 et 2010, lance quatre appels pour des projets «biomasse». Cette brutale montée en puissance de la politique énergétique et la perspective particulière de solliciter les ressources forestières avec des équipements imposants, subventionnés et dédiés à la production d'électricité, suscitent une forte réaction de la part de l'industrie papetière (Alexandre et al., 2012). Celle-ci dénonce une grave distorsion de concurrence favorisant les nouveaux entrants du secteur de l'énergie au détriment du tissu industriel existant et de l'emploi. Mais ce travail politique vise finalement moins à contester la légitimité des nouveaux projets «biomasse» qu'à réorienter les conditions de leur mise en œuvre pour transformer la menace en levier de développement (Montouroy et Sergent, 2014). L'industrie papetière voit en effet dans les dispositifs de soutien aux EnR une opportunité pour sécuriser ses investissements, collaborer avec des énergéticiens, diversifier ses débouchés et in fine faire évoluer son modèle industriel vers le paradigme des bioraffineries (Näyhä et Pesonen, 2014). Pour défendre cette mutation, elle met en avant sa capacité à être un moteur d'une «croissance verte» qui nécessite de mobiliser davantage de bois tout en limitant le gaspillage d'une ressource productrice de valeur ajoutée. Appuyés par des groupes de pression industriels et environnementaux, les papetiers obtiennent notamment de l'Union

${ }^{7}$ La productivité du pin maritime est passée de 4,8 à $7,8 \mathrm{~m}^{3}$ de bois par hectare et par an entre 1960 et 1978 (Lesgourgues et Drouineau, 2009). 
européenne et de l'État un renforcement de l'efficacité énergétique des projets sélectionnés (Montouroy et Sergent, 2014). Sont ainsi privilégiés les procédés de cogénération permettant de valoriser l'électricité et la chaleur produite, ce qui correspond parfaitement au profil des papetiers ${ }^{8}$. En 2006, les trois usines situées sur le massif landais répondent au deuxième appel d'offre de la CRE et l'une d'elle ambitionne même d'abriter la centrale de cogénération la plus puissante de France (69 mégawatt d'électricité, soit une consommation annuelle d'environ 500000 tonnes de BE).

Tout en se positionnant sur les nouveaux marchés de l'énergie, les papetiers landais continuent à problématiser le développement du $\mathrm{BE}$ comme un facteur de déstabilisation collective. L'enjeu est tout autant d'éviter l'implantation d'autres industriels que de verrouiller la filière locale d'approvisionnement. C'est ainsi qu'en 2007, le monde de la trituration s'oppose à l'installation d'une usine de granulés (combustible bois compacté) sur le port de Bayonne en affirmant notamment que les scieurs eux-mêmes pourraient pâtir de ce projet. Pourtant, les scieurs sont, a priori, les premiers bénéficiaires d'une activité valorisant prioritairement leurs produits connexes. Dans la presse régionale, les fabricants de panneaux et les papetiers exposent un tout autre point de vue: "Si nous ne pouvons plus trouver de produits connexes pour nos usines, nous achèterons alors directement des grumes ${ }^{9} »$, c'est-à-dire la matière première des scieurs. Cette menace explicite suggère qu'en répondant aux appels d'offre de la CRE, les acteurs de la trituration ont paradoxalement affaibli leur capacité à s'ériger en " porte-paroles » du système sociotechnique landais. «En faisant voir ce qui n'avait pas lieu d'être vu et en faisant entendre comme discours ce qui n'était entendu que comme bruit» (Rancière, 1995), leur stratégie duale ouvre en effet la voie à quelques «procès d'accusation » (Akrich et al., 1988) : «Ce sont les mêmes qui mettent plusieurs centaines de milliers de tonnes dans leurs chaudières qui nous accusent de déstructurer le marché avec nos projets à quelques centaines de tonnes » (Collectivité territoriale, 2009); «Ce double discours fut très irritant. Qu'ils veuillent couper l'herbe sous le pied de tout le monde, c'est de bonne guerre mais pour protéger leurs projets, ils voulaient qu'on protège la ressource et ça ce n'est pas bien » (Propriétaire 1, 2012).

C'est dans ce contexte de fragilisation des équilibres locaux que resurgissent les souches. Pour défendre leur positionnement, les papetiers affichent en effet leur ambition de mobiliser des ressources non conflictuelles : «Contrairement aux opérateurs qui arrivent avec le seul

\footnotetext{
${ }^{8}$ Sept des dix centrales installées suite aux deux premiers appels d'offre de la CRE se situent chez des papetiers (Carrier et al., 2015).

${ }^{9}$ Aquitaine presse service, mai 2007.
}

objectif de brûler du bois, nous, on est capable de s'arbitrer par rapport aux conséquences du BE sur notre process et sur l'ensemble de la filière. C'est bien pourquoi on a choisi d'investir sur les souches » (Industriel 1，2012). Grâce au projet européen «Forenergy», les papetiers landais savent en effet depuis 2001 que leurs homologues finlandais ont réussi à stabiliser un processus de valorisation énergétique des souches d'épicéa en développant une technologie adaptée: les chaudières à «lit fluidisé bouillonnant» (Hakkila, 2006). Lorsque l'opportunité des appels d'offre de la CRE se présente, les papetiers landais renouvellent donc leur équipement en optant pour cette technologie qui présente le grand intérêt de pouvoir traiter des combustibles hétérogènes et de médiocre qualité (Bonaiti, 2000). Ce faisant, les papetiers landais se mettent en capacité de résoudre une partie des écueils rencontrés dans les années 1970; mais une partie seulement.

\section{Les tempêtes, auxiliaires efficaces de la construction d'une ressource}

En intégrant dès 2007 les souches dans les plans d'approvisionnement de leurs nouvelles chaudières, les papetiers landais prennent un risque. Certes, ils se sont équipés technologiquement et peuvent s'appuyer sur l'expérience scandinave. Mais le marché n'existe pas et ils doivent encore convaincre leurs partenaires et plus particulièrement les propriétaires du bien-fondé de leur démarche. Les tempêtes Martin (1999) et surtout Klaus (2009) vont activement contribuer à cette entreprise en faisant converger l'ensemble des protagonistes autour du scénario proposé.

\section{La rareté de la ressource, un argument d'autorité pour en explorer de nouvelles}

Les tensions sur la ressource sont au cœur de l'argumentaire développé par les papetiers pour justifier le recours aux souches. Bien que martelée dans différentes arènes, cette rhétorique est contrecarrée, au milieu des années 2000, par l'idée prédominante d'une ressource forestière abondante à l'échelle nationale. Suite à un inventaire réalisé en 2005, l'ADEME estime ainsi que, "sans créer de concurrence avec les industries de la trituration, il existe une ressource de bois mobilisable et utilisable » (IFN, 2005). Dans cette étude, qui donne lieu à une publication au titre retentissant «Bois-énergie: les forêts ont de la ressource!», l'Aquitaine est même présentée comme la «Région au plus gros potentiel». Or, la tempête Klaus modifie radicalement ce discours ambiant. En effet, à partir de 2009, « la question de la ressource forestière dans les Landes hante tous les esprits [...]. L'enjeu est lourd: derrière la forêt, il y a une industrie et ses quelque 30000 
emplois qu'il faut continuer à faire tourner. Les chiffres sont implacables ${ }^{10} »$. Ces chiffres, ce sont ceux d'un volume de bois sur pied divisé par deux en dix ans et, en corollaire immédiat, l'annonce d'un «trou de production », «de l'ordre de $38 \%$ à $48 \%$ à l'horizon $2015 »$ (GIP Ecofor, 2010). Alors même que le processus de «traduction» qui permet de transformer les dégâts de la tempête en «trou de production» repose sur certaines hypothèses discutables ${ }^{11}$, les preuves quantifiées de la rareté à venir tendent à saturer l'espace public de sentiments consensuels quant à la nécessité de sécuriser l'approvisionnement du tissu industriel. En 2010, dans un article intitulé «Les industriels accusent les énergéticiens de pillage $»^{12}$, des acteurs de la puissance publique s'associent ainsi au monde de la trituration pour dénoncer les «nouveaux venus qui cherchent à s'insérer dans le paysage ». Cette mobilisation trouve son aboutissement dans la décision prise par les représentants de l'État en Aquitaine de restreindre l'accès au massif: «De la comparaison des données et des simulations, il ressort qu'un développement du BE sur la ressource "pin maritime" n'est pas envisageable ${ }^{13} »$.

Le «trou de production» annoncé ne permet pas seulement de «raisonner le développement du BE» (GIP Ecofor, 2010), il légitime également l'exploration de nouvelles ressources (Bridge et Wood, 2010; Mitchell, 2011). Dans cette perspective, plusieurs options sont avancées, dont «l'extension de la forêt de pin maritime, notamment en Dordogne, comme alternative au taillis de châtaignier dégradé » (Lesgourgues et Drouineau, 2009) ou encore le «déstockage des vieux bois » (GIP Ecofor, 2010 ; Thivolle-Cazat, 2013). Mais, tandis que la transformation du Périgord en nouvelle zone d'expansion de la forêt landaise constitue une entreprise de longue haleine, la proposition de mobiliser

\footnotetext{
${ }^{10}$ Sud-Ouest, janvier 2013.

${ }^{11} \mathrm{En}$ ce qui concerne l'offre, les experts eux-mêmes reconnaissent de nombreuses incertitudes tant dans l'évaluation de la ressource sur pied (âge des peuplements, etc.) que sur les options de gestion qui prévaudront (GIP Ecofor, 2010). Or, ces facteurs influencent la quantité et le type de bois mobilisables. En ce qui concerne la demande, les experts ont construit leur hypothèse en se basant notamment sur le constat d'une augmentation de $22 \%$ en 10 ans de la consommation de BI. Cette hypothèse haute ne tient donc pas compte de la crise économique et de la mauvaise santé d'une des trois usines papetières du massif. Enfin, l'expertise considère que la demande en BE devrait passer de 460000 à 2 millions de tonnes entre 2009 et 2015 . Or, à l'époque, la plupart des observateurs s'alarment de l'absence d'informations fiables et notamment de l'écart existant entre les projets annoncés et ceux réalisés (Alexandre et al., 2012 ; Poupeau et Schlosser, 2010).

${ }^{12}$ Sud-Ouest, avril 2010.

${ }^{13}$ Cellule Biomasse régionale, note stratégique 2010.
}

des qualités de bois traditionnellement destinés aux marchés du BO suscite une forte réaction du président du Syndicat des Sylviculteurs du Sud-Ouest (SYSSO): «Le discours récurrent sur le manque de ressource comporte une grosse part d'interprétation [...]. En fait, nous disposons de quinze ans d'approvisionnement des scieries [...]. Ce discours émane essentiellement des industriels pour se prémunir de tout nouvel investissement sur le massif ${ }^{14}$.» Cette réaction pourrait laisser penser que les rapports de force entre le monde de la trituration et les propriétaires ont peu évolué depuis les années 1970. Mais la proposition même de «déstocker les vieux bois » témoigne et participe d'un processus continu de dépréciation des qualités $\mathrm{BO}$ du pin maritime $^{15}$ qui, conjugué au déclin de ce secteur d'activité (Lévy et Belis-Bergouignan, 2011) et à la répétition des tempêtes, a largement déplacé les espaces de compromis envisageables. La perspective de raccourcir les cycles de rotation du pin maritime ne choque ainsi plus grand monde, notamment parce qu'elle permettrait de réduire l'exposition du capital aux risques. Combinées au développement du BE, les tempêtes ont indéniablement accéléré et catalysé le travail de problématisation porté par les industries de la trituration autour d'une nécessaire intensification de la gestion forestière. Elles ont contribué à rendre possibles des formes d'exploitations sylvicoles difficilement concevables auparavant. L'hypothèse d'une valorisation des souches est ainsi devenue crédible, voire même incontournable, parce qu'à la différence des autres options, qui impliquent une transformation des modes de gestion, cette nouvelle ressource offrait l'avantage d'avoir été rendue accessible par les tempêtes.

\section{Du laboratoire grandeur nature à la mise en marché des souches}

Une partie des procédés de transformation des souches a été codifiée après le passage de la tempête Martin en 1999. À l'époque, la principale préoccupation des opérateurs et des propriétaires est de nettoyer les parcelles parsemées de souches renversées en essayant de réduire les coûts de reboisements et les risques phytosanitaires. Les chantiers sont à mis profit pour éprouver différentes techniques, avec pour résultat de déqualifier certaines pratiques usuelles, telles que le brûlage et l'enfouissement, et la promotion des opérations de «mise en cordons». Or, initialement défendu au nom de son intérêt écologique et économique,

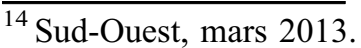

15 « L'idée d'un pin maritime qui ne vaut rien s'est complètement banalisée ! Ils le disent tous : “ça résine, ça tord, ça éclate ! Bon, on vous le prend quand même, mais pas cher !" » (Propriétaire 2, 2010).
} 
(a)

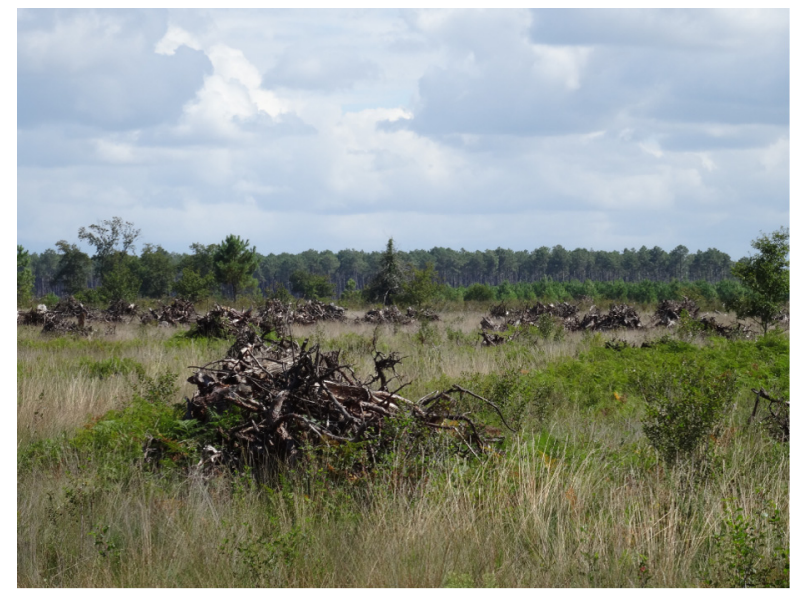

(b)

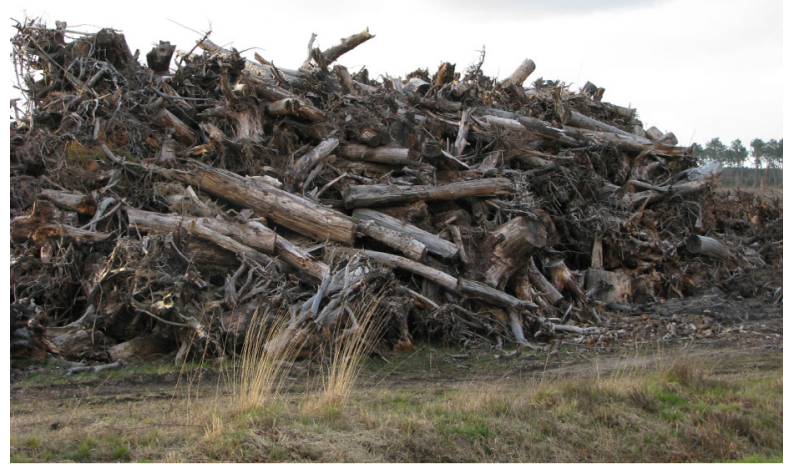

(c)

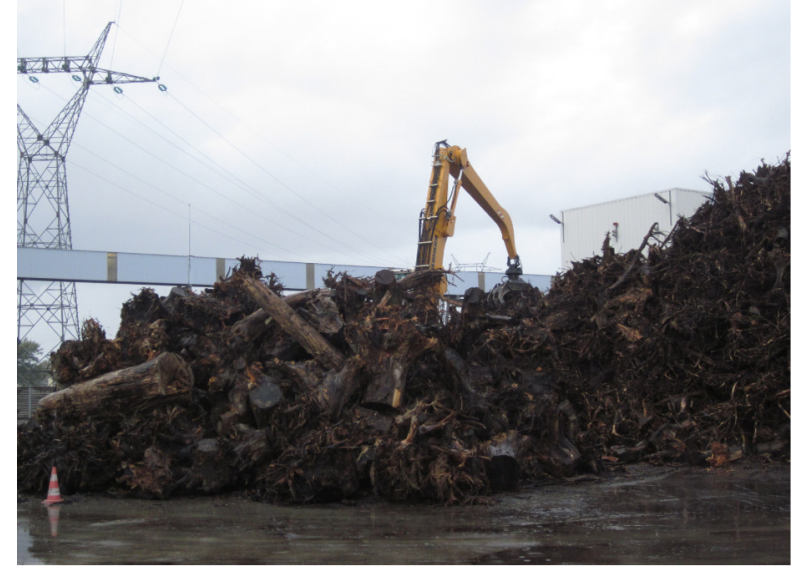

Fig. 2. Itinéraire d'une souche, de la parcelle (a) à la centrale de cogénération (c) en passant par le stockage en bord de route (b).

Sources : J. Dehez (a et b), V. Banos (c), 2013.

ce procédé consistant à aligner en bandes espacées des souches entières ou fragmentées est réapproprié en 2009 pour récupérer la biomasse dans la perspective d'un usage énergétique (Lesgourgues et Drouineau, 2009). Il permet, à un moindre coût, de stocker, de faire lessiver (par les intempéries) et donc de dessabler les souches (Fig. 2). Les réseaux d'expérimentations mis en place en
1999 servent ainsi d'appui aux programmes de recherche impulsés par les papetiers au moment où ces derniers se positionnent sur les appels d'offre de la CRE. Le projet «Bio-raffinerie et exploitation des forêts » (2006-2008) vise, par exemple, à concevoir un prototype, le «Rhino-Croq», adapté aux exigences du milieu landais : «En 1999, le matériel scandinave utilisé n'allait pas. Leurs souches d'épicéa sont plus petites avec pas mal de cailloux car c'est des anciennes moraines. Du coup, ils sont plus sur une technique de fendage. Avec le sable et nos racines, on a plutôt mis au point un outil de double cisaillement qui permet d'accélérer et de simplifier le process » (Opérateur 1, 2013).

À l'aube de la tempête de 2009, la chaîne d'approvisionnement est à peu près en place, du matériel d'extraction aux centrales de cogénération en passant par les dispositifs de stockage. Cependant, le gisement issu de la tempête Martin s'est émietté et les propriétaires ne sont pas encore prêts à laisser les souches issues des coupes rases. Mais, en plus de modifier l'état d'esprit des détenteurs de la ressource, la tempête Klaus va libérer une nouvelle quantité importante de matière et permettre aux papetiers d'amorcer le processus de commercialisation.

Les souches étant à nouveau transformées en «problème », les industriels peuvent enclencher la transaction sur le mode du service rendu. Cette posture peut être résumée ainsi : «Si nous proposons aux propriétaires de les débarrasser des déchets, nous facilitons et réduisons les coûts d'une tâche à laquelle ils ne peuvent se dérober (le reboisement). Ils ne peuvent que nous en être reconnaissants » (Akrich, 1989). En jouant aussi sur le fait que les travaux de nettoyage sont subventionnés, certains acheteurs parviennent ainsi à capter gratuitement ce gisement, avant que d'autres ne proposent le prix de $2 € /$ T(Alonso, 2013). L'extrait suivant synthétise les mécanismes de ce prix « symbolique » qui intègre les dispositifs d'intéressement élaborés dans les années 1970 :

« $2 € / T$, c'est pour dédommager le propriétaire qui garde sur son terrain le lot acheté. Mais là où il y a un gain réel pour lui, c'est au niveau de la protection sanitaire et du reboisement car un sol dessouché permet de meilleurs rendements et une meilleure croissance des plants [...]. Si le propriétaire doit y trouver son intérêt, il faut aussi qu'il se rende compte des efforts fournis pour traiter cette matière qui ne valait rien et qui reste coûteuse à produire. Si on me demande $10 € / T$, je laisse la souche sur place» (Opérateur 2, 2013).

Les propriétaires enquêtés reconnaissent l'attrait de ces arguments et ce d'autant plus que le Fomes - ce champignon se nourrissant du pourrissement des souches - prolifère dans une forêt fragilisée par deux tempêtes successives (Alonso, 2013). Ils soulignent plus globalement leur faible marge de manœuvre, pris entre l'obligation de sortir les souches et le peu d'usines 
capables de les consommer. Mais, après un temps de flottement, les organisations professionnelles réagissent. En 2012, elles signent une « feuille de route » qui rejette l'idée d'un "bois fatal non marchand » et réclame, entre autres, l'établissement de contrats d'approvisionnement et un «juste prix». Dans la continuité, un "Mémento aquitain du bois énergie » (CRPF, 2013) est rédigé afin de diffuser aux producteurs un certain nombre d'informations techniques et commerciales. En 2014, le SYSSO mobilise des arguments juridiques pour expliquer que la souche ne saurait être considérée comme un simple prolongement de l'arbre et que son acquisition doit faire l'objet d'une contractualisation spécifique. Il préconise aussi un prix minimum de $10 € / \mathrm{T}^{16}$. Certains propriétaires vont plus loin et demandent un montant compris entre 20 et $25 € / T$ en considérant que la valeur de la souche ne doit pas être indexée sur ses coûts de production mais sur les prix du pétrole et la substitution énergétique qu'elle permet. Même si cette position est plus marginale, elle montre bien les luttes définitionnelles à l'œuvre autour des qualités de la souche et la manière dont celles-ci jouent sur les transactions monétaires proposées. Mais ces débats s'inscrivent aussi dans des logiques de négociation qui, au final, témoignent d'un certain processus de «normalisation» des souches en tant que coproduits de l'exploitation forestière (Fig. 2).

Dans l'optique d'une telle standardisation, la tempête Klaus a également fait gagner du temps. Les expérimentations ont pu se poursuivre et ont contribué à stabiliser certaines controverses. Alors que la fertilité des sols constitue une préoccupation majeure pour des sylviculteurs landais confrontés à «un écosystème forestier très pauvre» (Lesgourgues et Drouineau, 2009), les scientifiques suggèrent ainsi que l'extraction des souches «pourrait avoir un impact limité en termes nutritionnels » (Augusto et al., 2014). Quant au bilan carbone, si son enjeu est soulevé par ces mêmes auteurs, force est de constater qu'il suscite encore peu de débats au sein du système sociotechnique. Entre les controverses désamorcées et celles qui peinent à émerger, les souches landaises ont ainsi poursuivi leur chemin vers la reconnaissance. En 2013, l'étude nationale RESOBIO, financée par l'ADEME et consacrée aux conséquences environnementales de la gestion des rémanents forestiers, entérine leur exploitation et envisage même une certification (Landmann et Nivet, 2013).

\section{Conclusion}

L'analyse $\mathrm{du}$ processus de transformation des souches du pin maritime en nouvelle ressource biomasse

\footnotetext{
${ }^{16}$ Forêt de Gascogne : Journal des sylviculteurs du Sud-Ouest, mai 2014.
}

montre que cette innovation a nécessité la mise en relation d'acteurs, d'entités et d'évènements disparates, des industries de la pétrochimie aux propriétaires forestiers, d'un champignon parasite aux chaudières à lits fluidisés, des appels d'offre de la CRE aux tempêtes. Elle met également en lumière les efforts déployés, entre négociation et coups de force, par les promoteurs du projet pour réorienter des politiques publiques, intéresser des partenaires réticents et transformer en alliés des entités initialement étrangères au système sociotechnique (les énergéticiens, les tempêtes, etc.). Malgré la force des liens préexistants et leur pouvoir de coordination, les papetiers ont en effet dû faire preuve de patience et naviguer au milieu d'incertitudes pour faire avancer leur innovation dans un monde recomposé par des politiques énergétiques tâtonnantes et des évènements imprévus. Ce processus erratique invite bien à relativiser, sans pour autant l'éluder, le rôle du développement technologique. Celui-ci n'est pas la solution déterminante qui a tout fait basculer mais une étape supplémentaire dans un travail d'assemblage ayant nécessité l'action non pas d'une mais de deux tempêtes majeures. De même, ce n'est pas la rentabilité intrinsèque des souches qui a guidé la démarche. Le recours à ce gisement inexploité s'apparente davantage à une démonstration permettant aux papetiers de légitimer une stratégie pertinente d'un point de vue économique mais délicate au niveau politique: se positionner sur les nouveaux marchés de l'énergie tout en faisant protéger «leurs» ressources usuelles en BI. Le fait que les bénéfices escomptés ne se situent pas tant dans les souches elles-mêmes mais soient plutôt une conséquence indirecte de cette valorisation (i.e. maintenir la profitabilité du BI) interroge d'ailleurs sur la pérennité de ce nouveau marché. L'exploitation généralisée des souches apparaît suspendue à la définition d'un compromis monétaire incertain entre des propriétaires, désireux $a$ minima d'amortir leurs investissements, et des consommateurs peu enclins à payer une ressource qui ne leur est pas indispensable.

Les rouages de ce processus d'innovation appellent enfin à revenir sur les potentialités associées à la transition énergétique. Ils dévoilent la construction d'une politique «locale» bois-énergie qui se traduit moins par une remise en partage des ressources forestières existantes que par le renforcement d'industries déjà bien installées et une intensification des pratiques sylvicoles. Cette dynamique de captation empreinte de logiques extractives (Bridge, 2010; Mitchell, 2011) remet en question l'idée selon laquelle les EnR seraient fondamentalement porteuses d'un changement de société, de nouvelles relations socioécologiques (Raineau, 2011). Mais elle invite à nuancer tout autant les théories du «carbon lock-in» (Unruh, 2000) qui tendent à enfermer un peu trop rapidement les systèmes sociotechniques 
hérités des énergies fossiles dans un rôle de simple barrière. En effet, si la construction de cette politique « locale» vise bien à maintenir un ordre établi, elle ne conduit pas pour autant à une reproduction de ce dernier. Tandis que les papetiers sont embarqués dans une trajectoire de «décarbonisation » et de mutation identitaire guidée par le paradigme de la «croissance verte», l'exploration de nouvelles ressources recompose le milieu associé à cette organisation industrielle ainsi que les liens entre acteurs. L'approche STS offre l'opportunité d'être attentif à ces déplacements et, finalement, aux multiples sentiers empruntés par la transition énergétique. Mais, comme nous l'avons esquissé dans cet article, les enjeux énergétiques confirment surtout l'intérêt de remettre les ressources et contraintes liées aux matérialités de la nature au cœur des processus de construction et de régulation des relations socioécologiques (Bakker et Bridge, 2006; Deshaies et Mérenne-Schoumaker, 2014 ; Kaup, 2008 ; Labussière et Nadai, 2015).

\section{Références}

ADISCO, 1983. Analyse technico-économique de la ressource supplémentaire mobilisable dans le massif Gascon. Rapport, Bordeaux, Association pour le développement des industries chimiques et pétrochimiques du Sud-Ouest.

Akrich M., 1989. La construction d'un système sociotechnique. Esquisse pour une anthropologie des techniques, Anthropologie et Sociétés, 13, 2, 31-51.

Akrich M., Callon M., Latour, B., 1988. À quoi tient le succès des innovations ? 1 : L'art de l'intéressement, Les Annales des Mines, 4-17.

Alexandre S., Gault J., Guerin A.-J., Lefebvre E., Menthière (de) C., Rathouis P., Texier P.-H., Thibault H.-L., Toussaint X., Attali C., 2012. Les usages non alimentaires de la biomasse, Rapport de mission, Paris, CGAAER.

Alonso A., 2013. Permanence et changements de pratiques chez les propriétaires forestiers landais après la tempête de 2009 : le bois énergie, une option?. Mémoire de Master 2, Bordeaux, Université Bordeaux Segalen.

Augusto L., Achat D., Bakker M., Boulanger V., Canteloup D., Landmann G., Trichet P., 2014. Intensification des itinéraires et des récoltes : implication pour la durabilité des systèmes, Innovations Agronomiques, 41, 13-30.

Bakker K., Bridge G., 2006. Material Worlds? Resource geographies and the "matter of nature", Progress in Human Geography, 30, 1, 5-27.

Banos V., Dehez J., 2015. Les trajectoires du bois-énergie en Aquitaine : du développement local aux territoires de l'énergie ?, Géocarrefour, 90, 4, 329-338.

Barbier R., Trepos J.-Y., 2007. Humains et non-humains : un bilan d'étape de la sociologie des collectifs, Revue d'anthropologie des connaissances, 1, 1, 35-58.

Barbier R., Nadai A., 2015. Acceptabilité sociale : partager l'embarras, VertigO, 15, 3, http://vertigo.revues.org/16686.
Bassett T.J., Peimer E.J., 2015. Political ecological perspectives on socioecological relations, Natures Sciences Sociétés, 23, 2, 157-165.

Benjaminsen T.A., Svarstad H., 2009. Qu'est-ce que la "political ecology"?, Natures Sciences Societés, 17, 1, $3-11$.

Bennett J., 2010. Vibrant Matter: A Political ecology of things, Durham, Duke University Press.

Bonaiti J-P., 2000. La combustion en lits fluidisés : émergence et hybridation d'une nouvelle technologie, in Bourgeois B., Finon D., Martin J-M (Eds), Énergie et changement technologique : une approche évolutionniste, Paris, Economica, 65-99.

Bridge G., 2010. Resource geographies 1: Making carbon economies, old and new, Progress in Human Geography, $35,6,820-834$.

Bridge G., Wood A., 2010. Less is more. Spectres of scarcity and the politics of resource access in the upstream oil sector, Geoforum, 41, 565-576.

Callon M., 1999. Pour une sociologie de la traduction en innovation : entretien avec R. Lhomme et J. Fleury, Recherche et Formation, 31, 113-126.

Callon M., 1986. Éléments pour une sociologie de la traduction. La domestication des coquilles Saint-Jacques dans la Baie de Saint-Brieuc, L'Année sociologique, 36, 169-208.

Callon M., 2009. La formulation marchande des biens, in F. Vatin (Ed.), Évaluer et valoriser : une sociologie économique de la mesure, Toulouse, Presses Universitaire du Mirail, 247-269.

Carrier T., Collet I., Louati S., Reynaud D., 2015. Chiffres clés des énergies renouvelables, Paris, Commissariat général au développement durable, http:/www.aitf.fr/system/files/ files/rep_-_chiffres_cles_energies_renouvelables.pdf.

Castree N., Braun B. (Eds.), 2001. Social nature: theory, practice and politics, Oxford (UK) and Malden MA, Blackwell Publishers, https://selforganizedseminar.files. wordpress.com/2011/08/castree_braun_social_nature.pdf.

Colin A., Barnérias C., Salis M., Coulon F., Couturier C., Thivolle-Cazat A., Rantien C., 2009. Biomasse forestière, populicole et bocagère disponible pour l'énergie à l'horizon 2020. Rapport final, ADEME, Angers, www. dispo-boisenergie.fr/doc/ADEME_boisenergie_rapport final_dec2009.pdf

CRPF, 2013. Mémento aquitain du bois-énergie (fiches de synthèse), www.crpfaquitaine.fr/documentation.php? Id theme $=4$.

Deshaies M., Mérenne-Schoumaker B., 2014. Ressources naturelles, matières premières et Géographie. L'exemple des ressources énergétiques et minières, Bulletin de la Société Géographique de Liège, 62, 53-61.

Fitzsimmons M., 1989. The matter of nature, Antipode, 21, 2 109-120.

Geels F.W., 2002. Technological transitions as evolutionary reconfiguration processes: toward a multi-level perspective and a case-study, Research Policy, 31, 8-9, 1257-1274.

GIP Ecofor, 2010. Pérennité de la ressource forestière et son adéquation avec les besoins industriels. Rapport 
d'expertise sur le devenir du massif des Landes de Gascogne et de la filière Pin maritime après la tempête de 2009, Bordeaux \& Paris, GIP Ecofor.

Gregori (de) T.R., 1987. Resources are not; they become: an institutional theory, Journal of Economic Issues, 21, 3, 1241-1263.

Hakkila P., 2006. Factors driving the development of forest energy in Finland, Biomass and Bioenergy, 30, 280-288.

IFN, 2005. Bois-énergie : les forêts ont de la ressource, L'IF, 9, http://inventaire-forestier.ign.fr/spip/IMG/pdf/L_IF_no09_ bois-energie.pdf.

Kaup B.Z, 2008. Negotiating through nature: the resistant materiality and materiality of resistance in Bolivia's natural gas sector, Geoforum, 39, 1734-1742.

Labussière O., Nadai A., 2015. L'énergie des Sciences Sociales, Paris, Alliance ATHENA.

Landmann G., Nivet C. (Coord.), 2013. Gestion des rémanents forestiers : préservation des sols et de la biodiversité. Rapport final du projet RESOBIO, ADEME, GIP Ecofor et Ministère de l'Agriculture, de l'Agroalimentaire et de la Forêt, www.ademe.fr/sites/default/files/assets/documents/ resobio-2-rapport-final-2014mars.pdf.

Lawhon M., Murphy J.T., 2011. Sociotechnical regimes and sustainability transitions: insights from political ecology, Progress in Human Geography, 36, 3, 354-378.

Lesgourgues Y., Drouineau S., 2009. Élaboration de nouveaux itinéraires techniques de régénération de la forêt landaise en réponse aux scénarios possibles, Innovations Agronomiques, 6, 101-112.

Lévy R., Belis-Bergouignan M.C., 2011. Quel développement pour une filière fondée sur le partage d'une ressource localisée ?, Revue d'Économie Régionale \& Urbaine, 3, 469-497.

MAAF, 2016. Programme National de la Forêt et du Bois 2016-2026, Ministère de l'Agriculture, de l'Agroalimentaire et de la Forêt, http://agriculture.gouv.fr/sites/minagri/ files/160307_plannational_foretbois_03.pdf.

Maugé J-P., Léonard J., 1969. La ligniculture du pin maritime, Revue Forestière Française, 21, 489-498.

Mitchell T., 2011. Carbon Democracy : le pouvoir politique à l'ère du pétrole, Paris, La Découverte.

Montouroy Y., Sergent A., 2014. Le jeu transcalaire des papetiers dans le cadre de la mise en œuvre de la politique " climat-énergie »: le cas de l'Aquitaine, Critique internationale, 1, 57-72.
Mora O., Banos V., 2014. La forêt des Landes de Gascogne : vecteur de liens ?, VertigO, 14, 1, http://vertigo.revues.org/ 14631.

Näyhä A., Pesonen H.-L., 2014. Strategic change in the forest Industry towards the biorefining business, Technological Forecasting and Social Change, 81, 259-271.

Perkins H.A., 2007. Ecologies of actor-networks and (non) social labor within the urban political economies of nature, Geoforum, 38, 6, 1152-116.

Poupeau F-M., Schlosser F., 2010. La régulation de la filière bois énergie dans les Ardennes françaises : jeux et enjeux autour de la gestion de l'information, Politique et Sociétés, 29, 2, 3-28.

Polge H., 1977. Chronique internationale : vers une utilisation plus complète des arbres, Revue forestière française, 29, 5, 417-419.

Raineau L., 2011. Vers une transition énergétique ?, Natures Sciences Sociétés, 19, 2, 133-143.

Rancière J., 1995. La mésentente, Paris, Galilée.

Riedacker A., Robin S., 1987. La consommation de boisénergie en France après le second choc pétrolier, Revue forestière française, 39, 2, 81-100.

Robbins P., 2004. Political Ecology: a critical introduction, Oxford, Blackwell publishing.

Robbins P., 2007. The lawn people: how grass, weeds, and chemicals make us who we are, Philadelphia, Temple University Press.

Rumpala Y., 2013. Formes alternatives de production énergétique et reconfigurations politiques. La sociologie des énergies alternatives comme étude des potentialités de réorganisation du collectif, Flux, 92, 2, 47-61.

Simiane (de) C., Bertrand J-L., Doubliez B., 1977. Extraction et récupération des souches, Annales de Mécanisation Forestière, 229-267.

Thivolle-Cazat A. (Coord.), 2013. Analyse prospective de la ressource forestière et des disponibilités en bois de la région Aquitaine à l'horizon 2025. Rapport final, FCBA, IGN, INRA, CRPF, http://inventaire-forestier.ign.fr/spip/ IMG/pdf/Rapport_final_Etude_disponibilites_Aquitaine. pdf.

Unruh G.C., 2000. Understanding carbon lock-in, Energy Policy, 28, 817-830.

Walmsley J.D., Godbold J.D., 2009. Stump harvesting for bioenergy - A review of the environmental impacts, Forestry, 83, 1, 17-38.

Citation de l'article : Banos V., Dehez J., 2017. Le bois-énergie dans la tempête, entre innovation et captation? Les nouvelles ressources de la forêt landaise. Nat. Sci. Soc. 25, 2, 122-133. 